\title{
Regeneration of phase unlocked serial multiplexed DPSK signals in a single phase sensitive amplifier
}

Guan, Pengyu; Da Ros, Francesco; Kjøller, Niels-Kristian; Hu, Hao; Røge, Kasper Meldgaard; Galili, Michael; Morioka, Toshio; Oxenløwe, Leif Katsuo

Published in:

Optical Fiber Communication Conference 2017

Link to article, DOI:

10.1364/OFC.2017.Th4l.5

Publication date:

2017

Document Version

Peer reviewed version

Link back to DTU Orbit

Citation (APA):

Guan, P., Da Ros, F., Kjøller, N-K., Hu, H., Røge, K. M., Galili, M., Morioka, T., \& Oxenløwe, L. K. (2017)

Regeneration of phase unlocked serial multiplexed DPSK signals in a single phase sensitive amplifier. In Optical Fiber Communication Conference 2017 (pp. 1-3). Optical Society of America (OSA).

https://doi.org/10.1364/OFC.2017.Th4l.5

\section{General rights}

Copyright and moral rights for the publications made accessible in the public portal are retained by the authors and/or other copyright owners and it is a condition of accessing publications that users recognise and abide by the legal requirements associated with these rights.

- Users may download and print one copy of any publication from the public portal for the purpose of private study or research.

- You may not further distribute the material or use it for any profit-making activity or commercial gain

- You may freely distribute the URL identifying the publication in the public portal 


\title{
Regeneration of Phase Unlocked Serial Multiplexed DPSK Signals in a Single Phase Sensitive Amplifier
}

\author{
P. Guan, F. Da Ros, N. K. Kjøller, H. Hu, K. M. Røge, M. Galili, T. Morioka, and L. K. Oxenløwe \\ DTU Fotonik, Technical University of Denmark, Ørsteds Plads, 343, Kgs. Lyngby, 2800, Denmark \\ pengu@fotonik.dtu.dk
}

\begin{abstract}
We demonstrate phase-regeneration of phase unlocked OTDM-DPSK serial signals in a single phase sensitive amplifier through optical cross-phase modulation. The BER of an $8 \times 10$ Gbit/s OTDM-DPSK signal is improved by 2 orders of magnitude.

OCIS codes: (060.4510) Optical communications; (060.4230) Multiplexing; (070.7145) Ultrafast processing.
\end{abstract}

\section{Introduction}

All-optical regeneration has attracted a lot of attention, since it may offer a significant increase in the overall capacity and transmission reach of fiber communication systems, without significant increases in cost or power consumption [1]. The phase sensitive amplifier (PSA) has emerged as a promising all-optical way to regenerate phase-encoded signals. It has been used for single channel regeneration of differential phase-shift keying (DPSK) and quadrature phase-shift keying (QPSK) signals [2]. However, to offer energy-efficiency, the regenerator must be compatible with multiplexing techniques, such as serial multiplexing based on increasing the symbol rate by interleaving low speed channels in the time domain, or the parallel multiplexing (WDM) scheme based on increasing the number of wavelength channels. In particular, the serial multi-channel approach with fewer wavelength channels, has the potential to reduce the cost per bit due to the reduced number of components, and the power consumption [3]. Furthermore, it has been shown that a WDM regenerator can be implemented using a single serial signal regenerator through optical Fourier transformations, which can be achieved by intermediately converting the parallel channels to a single serial channel, and then applying serial channel regeneration, followed by serial-to-parallel conversion. In this way, 16 WDM channels were successfully regenerated in a single PSA [4]. It is therefore important to investigate PSA schemes for regeneration of serial multiplexed signals. The main challenge lies in aligning the phase of individual serial-channels at the PSA input.

In this paper, we demonstrate phase regeneration of serial multiplexed DPSK signals in a single PSA through optical cross-phase modulation (XPM). We simultaneously regenerate 8 phase noise degraded $10 \mathrm{Gbit} / \mathrm{s}$ DPSK serial channels in a single PSA. The BER performance is characterized focusing on the positioning of the regenerator, either directly in front of the receiver or between two broadband phase noise emulators (in-line regeneration). In this latter case more than 2 orders of magnitude improvement is achieved.

\section{Principle}

A serial multiplexed signal has the advantage of its single-wavelength feature, which enables regeneration of many serial channels using a single dual-pump PSA. However, the random carrier phase of the individual serial channels poses a challenge to phase sensitive optical processing. The main idea is to convert the serial multiplexed DPSK signal to a phase coherent (PC) one using a delay interferometer (DI) and XPM. The PC serial signal tributaries can then be regenerated simultaneously in a single PSA. The principle of all-optical serial multi-channel regeneration of phase unlocked DPSK signals is shown in Fig. 1. A serial multiplexed DPSK signal is generated by time interleaving a number of low symbol rate DPSK channels at the same wavelength with random carrier phase (A). A 1-bit DI at the base symbol rate is used to convert the DPSK signals to OOK signals, and the phase noise is converted to amplitude noise (B). XPM in a highly nonlinear fiber (HNLF) is employed to transfer the data modulation from the OOK signal to the phase of a continuous wave $(\mathrm{CW})$ carrier $(\mathbf{C})$, resulting in a serial PC-DPSK

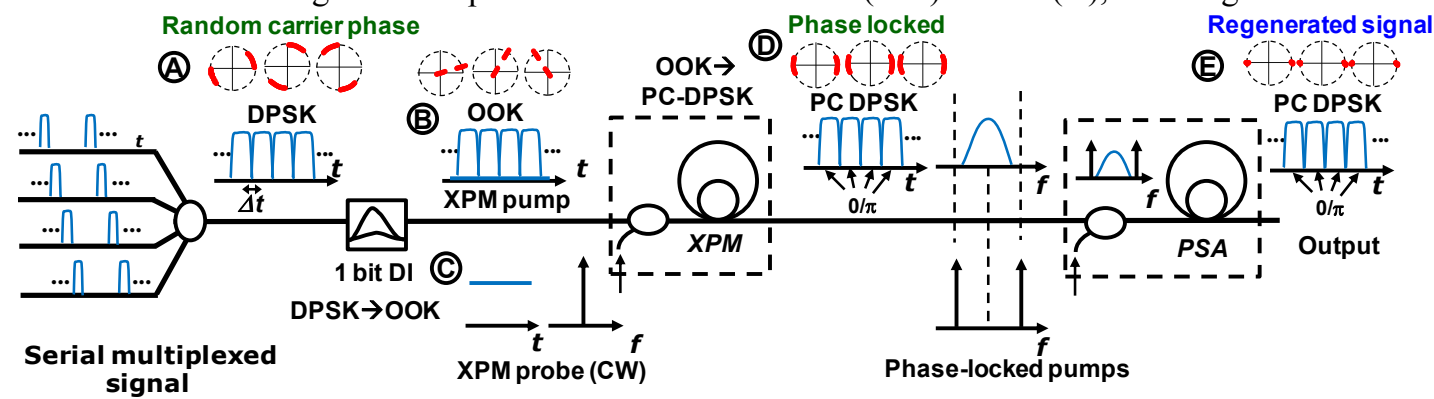

Fig. 1. Principle of all-optical serial multi-channel regeneration of phase unlocked DPSK signals. 


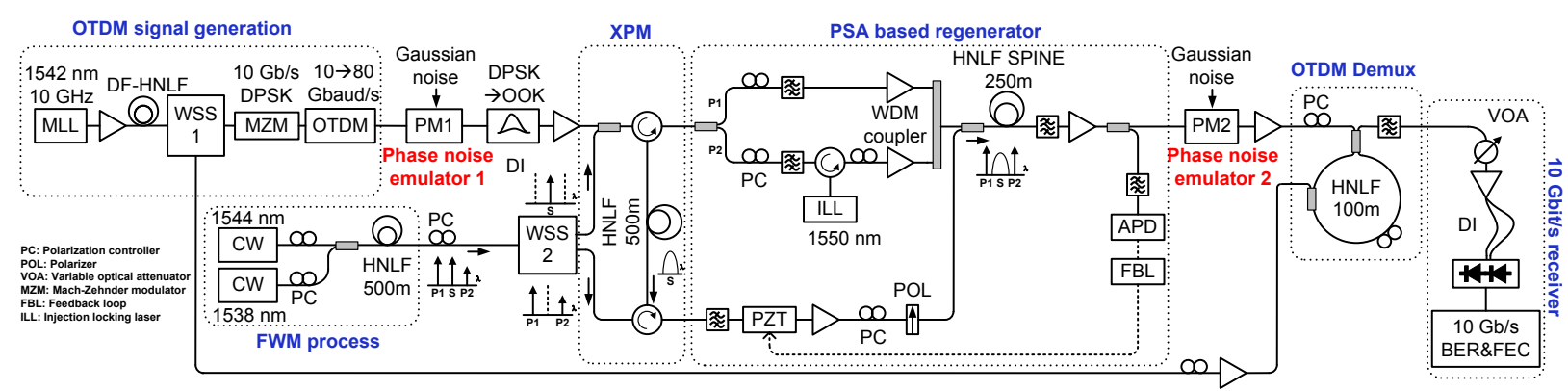

Fig. 2. Experimental setup of $8 \times 10$-Gbit/s serial multi-channel DPSK signal regeneration with two phase noise emulation stages.

signal (D). After combination with two phase locked pumps, the obtained PC-DPSK signal is sent to a HNLF-based PSA, resulting in a phase regenerated serial DPSK signal (E). The proposed scheme is scalable, since in principle the system complexity does not increase with the number of tributaries.

\section{Experimental setup and results}

In this experimental demonstration, an $8 \times 10 \mathrm{Gbit} / \mathrm{s}$ serial multiplexed DPSK signal is generated by optical time division multiplexing (OTDM). The experimental setup is shown in Fig. 2. A supercontinuum signal is generated by a 10-GHz mode-locked laser (MLL) and a 400-m dispersion-flattened HNLF (DF-HNLF). The broadened spectrum is sinc-filtered at $1562 \mathrm{~nm}$ by a programmable wavelength selective switch (WSS), resulting in a $12.5 \mathrm{ps}$ rectangular pulse train. After modulation, the 10 -Gbaud DPSK signal is then $8 \times$ OTDM-multiplexed using a fiber-based multiplexer, providing an 80-Gbit/s DPSK-OTDM signal with unlocked carrier phases for the different tributaries. As introduced before, the regenerator consists first of a DPSK-to-OOK conversion stage using a 1-bit (100 ps) DI. The waveform of the 80 Gbaud OTDM-OOK signal is shown in Fig. 3(a).

The three phase-locked carriers P1 $(1538 \mathrm{~nm}), \mathrm{S}(1544 \mathrm{~nm})$ and P2 $(1550 \mathrm{~nm})$ to be used for the PSA stage are generated based on four wave mixing (FWM) in a $500 \mathrm{~m}$ HNLF. The signal carrier is separated from the pumps by WSS2, and they are sent into a 500-m HNLF in counter-propagating directions by using optical circulators. The obtained OTDM-OOK signal is coupled into the HNLF to function as an XPM pump co-propagating with S. By carefully adjusting the pump power, XPM will impart BPSK modulation on the carrier S (Fig. 3b). Before the PSA, the pumps P1 and P2 are further split for independent amplification and an injection locking laser (ILL) is used to increase the OSNR of carrier P2, which is the idler of the FWM process. Signal and pumps are then launched into the PSA consisting of a $250 \mathrm{~m}$ HNLF with stable phase-matching for improved nonlinear efficiency (HNLFSPINE). The input power for $\mathrm{S}$ is $0 \mathrm{dBm}$ and $21 \mathrm{dBm}$ for $\mathrm{P} 1$ and $\mathrm{P} 2$. For active phase locking, $10 \%$ of the signal power was detected by a slow-speed avalanche photodiode (APD) after a narrow optical filter for a feedback loop (FBL) based on a piezoelectric actuator (PZT) [5]. The PSA output spectra for maximum and minimum output signal power are reported in Fig. 3(c), showing a dynamic phase sensitive extinction ratio of $8.2 \mathrm{~dB}$. In the receiver, the regenerated OTDM signal is demultiplexed with a nonlinear optical loop mirror using a $1.3 \mathrm{ps}$ wide gating pulse. Finally, the BER of each channel is measured in a 10-Gbit/s receiver including a DI and balanced photo-detection.

In order to test the regenerator, phase noise is added by up to two broadband phase noise emulators inserted both before and after the regenerator. Each phase noise emulator is based on a phase modulator (PM) driven by random noise with approximately Gaussian distribution obtained from an optical amplified spontaneous emission (ASE) source followed by a photodetector. The regeneration performance is investigated under two conditions: a single phase noise emulator with the regenerator directly in front of the receiver (Fig. 4(b)) and the regenerator in between two phase noise emulators, i.e. emulating the positioning of the regenerator within a transmission link (Fig 4(c)). The phase noise is quantified by the variance of optical phase, estimated by $D i=\left(\pi \cdot \sigma_{\mathrm{e}}^{\mathrm{i}} / V_{\pi}^{\mathrm{j}}\right)^{2}$, with $\sigma_{\mathrm{e}}^{\mathrm{i}}$ being the
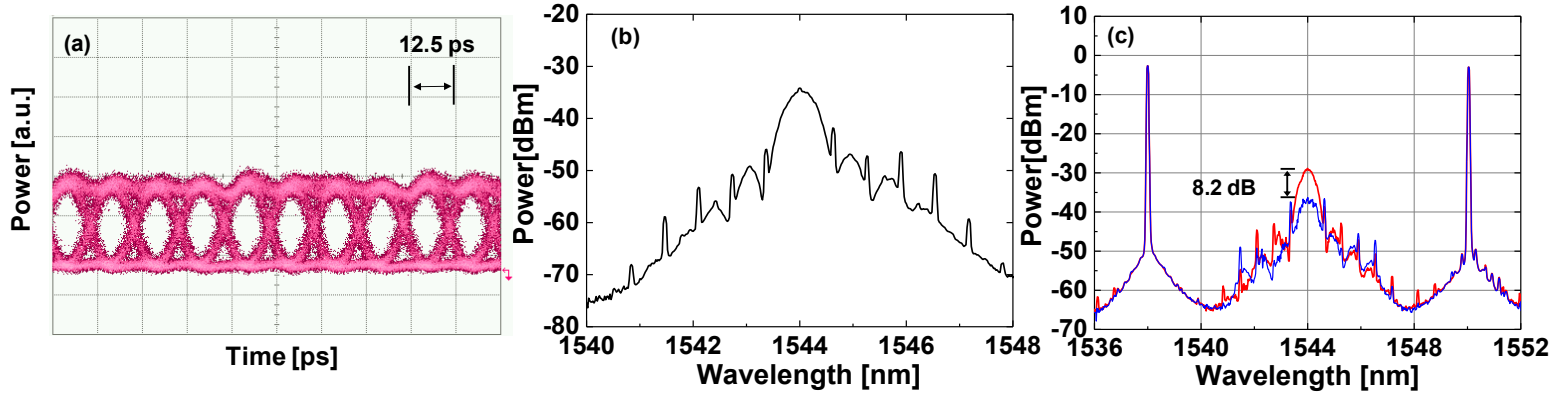

Fig. 3. (a) Waveform of the obtained 80 Gbaud OTDM-OOK signal. (b) optical spectrum of the XPM output, (c) spectra at the PSA output for maximum (red) and minimum (blue) output signal power. 

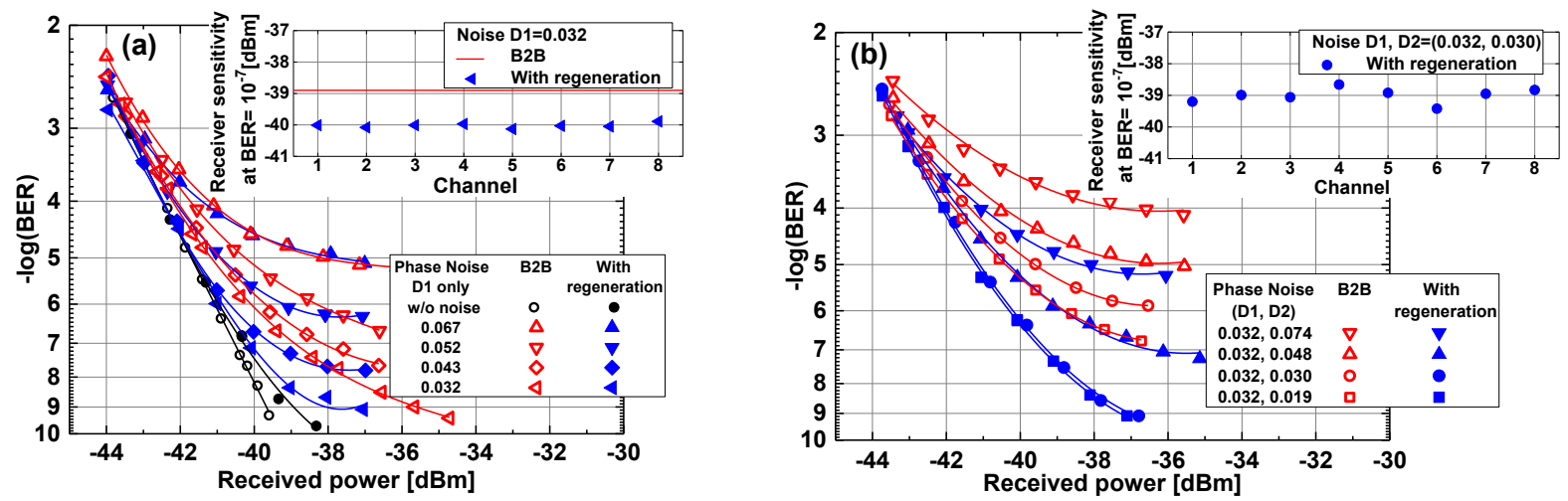

Fig. 4. Experimental results, (a) BER performance of the regenerated $80-\mathrm{Gbit} / \mathrm{s}$ DPSK serial channels with only one phase noise emulator (D1), inset: receiver sensitivities of all regenerated channels at BER $=10^{-7}$ with noise $\mathrm{D} 1=0.032$. (b) BER performance of the regenerated 80-Gbit/s DPSK serial channels with dual phase noise emulators (D1, D2), inset: receiver sensitivities of all regenerated channels at $\mathrm{BER}=10^{-7}$ with noise $(0.032,0.030)$.

standard deviation of the electrical driving voltage to the PMi and $V_{\pi}^{i}$ being the half-wave voltage of the PMi. Starting from the first case (regenerator in front of the receiver), Fig. 4(a) shows the BER performance of one demultiplexed 10-Gbit/s serial channel for different levels of phase noise, emulated from only PM1. The regenerator is benchmarked against the back-to-back (B2B) BER curves with and without phase noise. The regenerator power penalty without noise is only $0.7 \mathrm{~dB}$ at $\mathrm{BER}=10^{-9}$. For the $\mathrm{B} 2 \mathrm{~B}$ signal, adding noise not only increases the power penalty, but also introduces error floors due to the statistical properties of the Gaussian noise. The improvement in power penalty provided by the regenerator decreases with the increasing variance of the noise. In addition, the error floors cannot be removed, since the PSA cannot correct errors (phase shifts $>\pi$ ). The inset of Fig. 4(a) shows the receiver sensitivities at $\mathrm{BER}=10^{-7}$ for all regenerated serial $(\mathrm{OTDM})$ channels with phase noise $\mathrm{D} 1=0.032$. A similar improvement of more than $1 \mathrm{~dB}$ in receiver sensitivity was achieved for all serial channels.

The case of the regenerator positioned within a transmission link is shown in Fig. 4 (b). The BER performance of the regenerated 80-Gbit/s DPSK serial signal is reported for different levels of phase noise, emulated using both PM1 and PM2. In order to test the maximum noise tolerance after regeneration, the noise from PM1 was kept constant, while the noise from PM2 was increased. Without regeneration, the error floors appear and scale up with D2. With regeneration in between the two noise emulators, significant BER improvements are achieved for all the noise levels considered. In particular, without regeneration there are error floors at BER $=10^{-6}$ and $10^{-7}$ for noise combination $(\mathrm{D} 1, \mathrm{D} 2)=(0.032,0.030)$ and $(0.032,0.019)$. With regeneration, both BER curves are improved by more than 2 orders of magnitude achieving error-free $\left(B E R<10^{-9}\right)$ performance. Even with the largest phase noise considered $(0.032,0.074)$, more than one order of magnitude improvement in BER is achieved. This indicates that the proposed serial multi-channel regeneration scheme has a strong potential for in-line applications. The receiver sensitivities of all regenerated channels at $\mathrm{BER}=10^{-7}$ with phase noise $\mathrm{D}=(0.032,0.030)$ are also shown in the inset of Fig. 4(b). The performance variation is $<1 \mathrm{~dB}$ and enables decreasing the error floor for all channels.

\section{Conclusion}

We have demonstrated phase-regeneration of serial multiplexed DPSK signals in a single phase sensitive amplifier. $8 \times 10$-Gbit/s phase noise degraded DPSK serial channels with random carrier phase were regenerated simultaneously. When noise was added both before and after the regenerator to emulate a transmission scenario, BER improvements by 2 orders of magnitude were achieved. In principle, the system complexity of the proposed scheme does not scale with the number of serial channels, making the scheme highly flexible and scalable with the symbol rate. These advantages make this technique highly suitable for the implementation of functionality enabling the scalable WDM phase regenerator.

Acknowledgements: Danish Research Council projects LENS-COM (ref. DFF-5054-00184), TOR (ref. no. 12127224), NANO-SPECs (DFF-4005-00558B), DNRF Centre project SPOC (ref. DNRF123), and OFS Denmark.

\section{References}

[1] A. D. Ellis et al., "Phase sensitive signal processing using semiconductor optical amplifiers," Proc. OFC, OW4C.1, (2013).

[2] F. Parmigiani, "Phase sensitive amplifiers and their applications," Proc. OFC, W4C1, (2015).

[3] L.K. Oxenløwe et al, "Silicon Photonics for Signal Processing of Tbit/s serial data signals," IEEE. JSTQE., 18, 996 (2012).

[4] P. Guan et al., "16 Channel WDM Regeneration in a Single Phase-Sensitive Amplifier through Optical Fourier Transformation," Proc. ECOC, PDP, Th3B3, (2016).

[5] F. Da Ros et al., "Phase regeneration of DPSK signals in a silicon waveguide with reverse-biased p-i-n junction," Opt. Express, 22, 5029, (2014). 